\title{
Equitable Partition of Graphs into Independent Sets and Cliques
}

\author{
Bruno Monteiro ${ }^{1}$, Vinicius F. dos Santos $^{1}$ \\ ${ }^{1}$ DCC - Universidade Federal de Minas Gerais (UFMG) \\ Belo Horizonte - MG - Brazil \\ \{monteirobruno, viniciussantos\}@dcc.ufmg.br
}

\begin{abstract}
A graph is $(k, l)$ if its vertex set can be partitioned into $k$ independent sets and $l$ cliques. Deciding if a graph is $(k, l)$ can be seen as a generalization of coloring, since deciding is a graph belongs to $(k, 0)$ corresponds to deciding if a graph is $k$-colorable. A coloring is equitable if the cardinalities of the color classes differ by at most 1 . In this paper, we generalize both the $(k, l)$ and the equitable coloring problems, by showing that deciding whether a given graph can be equitably partitioned into $k$ independent sets and $l$ cliques is solvable in polynomial time if $\max (k, l) \leq 2$, and NP-complete otherwise.
\end{abstract}

\section{Introduction}

The Vertex Coloring Problem (VCP) consists of partitioning the vertex set of a graph into $k$ disjoint sets, such that no edges has both endpoints in the same set. This problem is known to be NP-complete [Karp 1972]. A widely studied variation of this problem is the Equitable Coloring Problem, in which we want the cardinalities of the sets to differ by at most 1 . The latter is also NP-Complete, since it can be reduced from VCP by adding sufficiently many isolated vertices.

Let $(k, l)$ be the set of graphs that can be partitioned into $k$ independent sets and $l$ cliques. Recognizing $(k, l)$ graphs can be seen as a generalization of the Equitable Coloring Problem (ECP), since deciding if a graph belongs to $(\boldsymbol{k}, \mathbf{0})$ corresponds to deciding if the graph is $k$-colorable. This problem was shown to be solvable in polynomial time if $\max (k, l) \leq 2$, and NP-complete otherwise [Brandstädt 1996, Brandstädt 1998, Brandstädt 1984].

In this paper we introduce the equitable version of the $(k, l)$ problem: we want the partition to be equitable. For that, let's define $(k, l)_{E}$ as the set of graphs that can be equitably partitioned into $k$ independent sets and $l$ cliques, that is, the cardinalities of the sets differ by at most 1 . It is easy to see that this is a generalization of ECP.

If $\bar{G}$ is the complement graph of $G$, then $G \in(k, l)_{E}$ if and only if $\bar{G} \in(l, k)_{E}$. Checking whether $G \in(\mathbf{1}, 0)_{E}$ is to check if $G$ has no edges, and to check whether $G \in$ $(0,1)_{E}$ is to check if $G$ is complete. Both can be easily done in linear time.

\section{Recognition of $(1,1)_{E}$ Graphs}

For a vertex $x \in V$, let $N(x)$ be the neighborhood of $x$ and $\bar{N}(x)$ be the set of nonneighbors of $x$. We will also denote $N[x]$ as $N(x) \cup x$ and $\bar{N}[x]$ as $\bar{N}(x) \cup x$.

Let $G=(V, E)$ be a graph, and $x \in V$ a vertex from $G$. We want to find an independent set $I$ and a clique $C$ such that $I \cup C=V$. If $x \in I$, then it does not have 
a neighbor in $I$. Therefore, $x \in I \Rightarrow N(x) \subseteq C$, that is, $N(x) \in(\mathbf{0}, \mathbf{1})$. Similarly, if $x \in C$, then it is neighbor with all other vertices in $C: x \in C \Rightarrow \bar{N}(x) \in(1,0)$. So we check, for every vertex $x$ from $G$, if $\mathbf{1}) N(x) \in(\mathbf{0}, \mathbf{1})$ and $\mathbf{2}) \bar{N}(x) \in(\mathbf{1}, \mathbf{0})$.

If there is a vertex that does not satisfy any of the previous conditions, then $G \notin$ $(1,1)_{E}$. If there is a vertex $x$ such that both 1 and 2 holds, then we can observe that $\bar{N}[x] \in \mathbf{( 1 , 0 )}$, and $N[x] \in(\mathbf{0}, \mathbf{1})$. Therefore, it is enough to insert $x$ in the set that has fewer elements, and we get the partitions $I=\bar{N}[x]$ and $C=N(x)$, or $I=\bar{N}(x)$ and $C=N[x]$. If the partition is equitable, then $G \in(\mathbf{1}, \mathbf{1})_{\boldsymbol{E}}$. Otherwise, $G \notin(\mathbf{1}, \mathbf{1})_{\boldsymbol{E}}$.

If every vertex satisfy exactly one of the conditions, we can construct the sets $I$ and $C$ in the following manner: for each vertex $x \in G$, if $x$ satisfies condition $\mathbf{1}$, insert it in $I$. if $x$ satisfies condition 2 , insert it in $C$. If at the end of the construction $I \in(\mathbf{1}, \mathbf{0})$ $\wedge C \in(\mathbf{0}, \mathbf{1}) \wedge|| I|-| C|| \leq 1$, then $G \in(\mathbf{1}, \mathbf{1})_{\boldsymbol{E}}$. Otherwise, $G \notin(\mathbf{1}, \mathbf{1})_{\boldsymbol{E}}$.

Checking conditions 1 and $\mathbf{2}$ can be easily done in $\mathcal{O}\left(|V|^{2}\right)$ time. Hence, deciding if $G \in(\mathbf{1}, \mathbf{1})_{\boldsymbol{E}}$ can be solved in $\mathcal{O}\left(|V|^{3}\right)$ time.

\section{Recognition of $(2,0)_{E}$ Graphs}

Deciding whether a graph $G=(V, E) \in(2,0)_{E}$ is the same as deciding if $G$ is equitably bipartite. For the sake of completeness we will prove this result here.

Verifying if $G \in(2,0)$ can be easily done in $\mathcal{O}\left(|V|^{2}\right)$. If $G$ is connected we check if the partition is equitable. Otherwise, we initially have two possibilities of partitioning for each connected component. In that case, we first find a bipartition for each of the $c$ connected components of $G$. If any of them is not bipartite, then $G \notin(2,0)_{E}$. Let $x_{i}$ and $y_{i}$ be the number of vertices in each of the sets from the partition of the $i$-th connected component of $G$.

We define the function $f(i, S)$, that equals TRUE if starting from the connected component with index $i$, there is a partition in two independent sets such that the number of vertices in one of the two sets is equal to $S$. Otherwise, $f(i, S)$ equals FALSE. Now we can notice that $f\left(1,\left\lfloor\frac{|V|}{2}\right\rfloor\right)$ answers if $G \in(\mathbf{2}, \mathbf{0})_{\boldsymbol{E}}$. This strategy is similar to the standard pseudo-polynomial algorithm for the Partition problem, with the difference that, in our case, numbers are bounded by the instance size.

Theorem 1. Deciding if $G=(V, E) \in(2,0)_{E}$ can be solved in $\mathcal{O}\left(|V|^{2}\right)$ time.

Proof. To prove that $f\left(1,\left\lfloor\frac{|V|}{2}\right\rfloor\right)$ can be computed in $\mathcal{O}\left(|V|^{2}\right)$ time, we will show that we only have to compute $\mathcal{O}\left(|V|^{2}\right)$ values of $f$, and that each of them can be computed in $\mathcal{O}(1)$ time, using previously computed values.

To actually compute $f(i, S)$, we check both possibilities of partition of the $i$-th connected component. Depending on which one we choose, the number of vertices we will need starting from the $(i+1)$-th connected component is either $S-x_{i}$ or $S-y_{i}$. The full algorithm to compute $f(i, S)$ is shown is Algorithm 1.

Since the values in the first parameter of $f$ varies from 1 to $c$, which is $\mathcal{O}(|V|)$, and the second parameter varies from $-\left\lceil\frac{|V|}{2}\right\rceil$ to $\left\lfloor\frac{|V|}{2}\right\rfloor$, which is also $\mathcal{O}(|V|)$, computing $f\left(1,\left\lfloor\frac{|V|}{2}\right\rfloor\right)$ depends on $\mathcal{O}\left(|V|^{2}\right)$ previous values of $f$. Moreover, the computation of $f$ takes $\mathcal{O}(1)$ assuming the values it depends on are already computed. Therefore, as long as values are not recalculated, computing $f\left(1,\left\lfloor\frac{|V|}{2}\right\rfloor\right)$ can be done in $\mathcal{O}\left(|V|^{2}\right)$ time. 


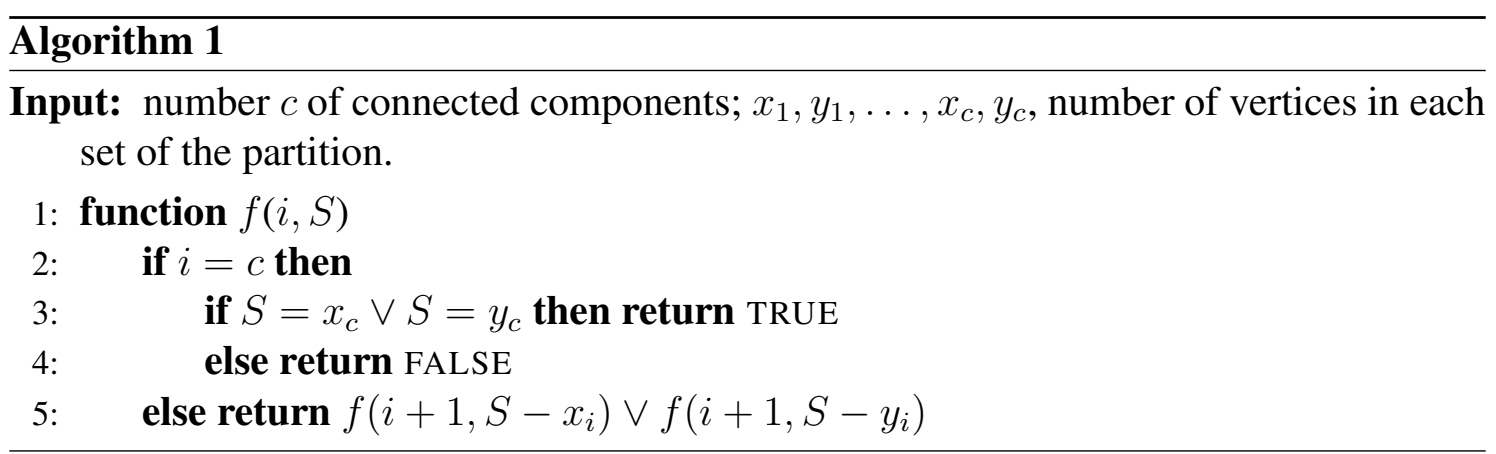

\section{Recognition of $(2,1)_{E}$ Graphs}

For the recognition of $(2,1)_{E}$ graphs, we will use an algorithm to find a $(2,1)$ partition of $G$. If $G \notin(2,1)$, then $G \notin(2,1)_{E}$. Let $I_{1}, I_{2}$, and $C$ be respectively the independent sets and the clique that partition $G$, such that $I_{1} \cup I_{2} \cup C=V(G)$. Let $I_{1}^{\prime}, I_{2}^{\prime}$, and $C^{\prime}$ be respectively the independent sets and the clique that equitably partition $G$. Since $I_{1}$ is an independent set, it is true that $\forall u, v \in I_{1},(u, v) \notin E(G)$. Therefore, at most one vertex of $I_{1}$ can belong to $C^{\prime}$, given that $C^{\prime}$ is a clique. Similarly, at most two vertices of $C$ can belong to $I_{1}^{\prime} \cup I_{2}^{\prime}$, each one in one of the sets.

Now, we look at all the possibilities to insert $I_{1}, I_{2}$, and $C$ in $I_{1}^{\prime}, I_{2}^{\prime}$, and $C^{\prime}$. For each possibility, we check if $I_{1}^{\prime} \cup I_{2}^{\prime} \in(2,0)_{E}$, if $C^{\prime} \in(0,1)$, and if the partition is equitable. If that is the case for any of the possibilities, then $G \in(2,1)_{E}$. Otherwise, $G \notin(2,1)_{E}$.

There are $\mathcal{O}(|V|)$ ways to choose one vertex from $I_{1}, \mathcal{O}(|V|)$ ways for $I_{2}$ and $\mathcal{O}\left(|V|^{2}\right)$ ways to choose two vertices from $C$. For every choice, checking whether $I_{1}^{\prime} \cup$ $I_{2}^{\prime} \in(2,0)_{E}$ can be done in $\mathcal{O}\left(|V|^{2}\right)$ time, from Theorem 1. It is trivial to check if $C^{\prime} \in$ $(\mathbf{0 , 1})$ in $\mathcal{O}\left(|V|^{2}\right)$. Hence, checking each of the $\mathcal{O}\left(|V|^{4}\right)$ possibilities is $\mathcal{O}\left(|V|^{2}\right)$, which gives a total of $\mathcal{O}\left(|V|^{6}\right)$. It is known that a $(2,1)$ partition of a graph can be found in $\mathcal{O}\left(|V|^{4}\right)$ time [Brandstädt 1996, Brandstädt 1998, Brandstädt 1984], so deciding if $G \in$ $(2,1)_{E}$ can be done in $\mathcal{O}\left(|V|^{6}\right)$ time.

\section{Recognition of $(2,2){ }_{E}$ Graphs}

We will use the same technique that was used in the $(2,1)_{E}$ recognition for the $(2,2)_{E}$ recognition. If $G \notin(2,2)$, then $G \notin(2,2)_{E}$. Now suppose that $G \in(2,2)$. Let $I_{1}, I_{2}$, $C_{1}$, and $C_{2}$ be respectively the independent sets and the cliques that equitably partition $G$.

Since $I_{1}$ is an independent set, then at most two of it's vertices can belong to $C_{1}^{\prime} \cup C_{2}^{\prime}$, each one in one of the cliques, and the same is true for $I_{2}$. Moreover, since $C_{1}$ is a clique, then at most two of it's vertices can belong to $I_{1}^{\prime} \cup I_{2}^{\prime}$, each one in one of the sets, and that also holds for $C_{2}$. So, there are $\mathcal{O}\left(|V|^{8}\right)$ possibilities of insertion in $I_{1}^{\prime}, I_{2}^{\prime}$, $C_{1}^{\prime}$, and $C_{2}^{\prime}$. If any of them is valid, that is, if they make an equitable partition of $G$, then $G \in(2,2)_{E}$. Otherwise, $G \notin(2,2)_{E}$.

For every one of the $\mathcal{O}\left(|V|^{8}\right)$ possibilities, we check if $I_{1}^{\prime} \cup I_{2}^{\prime} \in(2,0)_{E}$, if $C_{1}^{\prime} \cup C_{2}^{\prime} \in(\mathbf{0}, 2)_{E}$ and if the partition is equitable. Those conditions can be verified in $\mathcal{O}\left(|V|^{2}\right)$, from Theorem 1. Thus, given a $(2,2)$ partition of $G$, we can answer if $G \in$ $(2,2)_{E}$ in $\mathcal{O}\left(|V|^{10}\right)$ time. 
The best known algorithm capable of finding a $(2,2)$ partition of a graph runs in $\mathcal{O}\left(|V|^{12}\right)$ [Brandstädt 1996, Brandstädt 1998, Brandstädt 1984]. Hence, we are limited by that complexity, and answering whether $G \in(2,2)_{E}$ can be done in $\mathcal{O}\left(|V|^{12}\right)$ time.

\section{6. $(k, l)_{E}$ recognition for other values of $k$ and $l$}

We will now show that $(\boldsymbol{k}, \boldsymbol{l})_{\boldsymbol{E}}$ recognition for $\max (k, l) \geq 3$ is NP-complete. For that, we will make a reduction from the problem EQUITABLE COLORING. It is known that deciding whether a given graph $G$ can be equitably colored with $k$ colors is NP-complete for $k \geq 3$.

Let's define the join of two graphs $G$ and $H$ as the graph obtained by the disjoint union of $G$ and $H$ and the addition of all edges between vertices of $G$ and $H$. It suffices to show that $(\boldsymbol{k}, \boldsymbol{l})_{\boldsymbol{E}}$ is NP-complete for $k \geq 3$, since that $G \in(\boldsymbol{k}, \boldsymbol{l})_{\boldsymbol{E}} \Leftrightarrow \bar{G} \in(\boldsymbol{l}, \boldsymbol{k})_{\boldsymbol{E}}$.

Theorem 2. Deciding if $G=(V, E) \in(\boldsymbol{k}, \boldsymbol{l})_{\boldsymbol{E}}$ for $\max (k, l) \geq 3$ is NP-complete.

Proof. Since checking independent sets and cliques can be easily done in $\mathcal{O}\left(|V|^{2}\right)$, the problem belongs to NP. An instance of the EQUITABLE COLORING receives a graph $G=(V, E)$ and an integer $k$, and outputs YES if $G$ can be equitably colored with $k$ colors. We may assume that $|V| \geq 2 k$, otherwise we can color $G$ in polynomial time by finding a maximum matching in the complement, and that $k$ divides $|V|$, by adding an isolated clique of size at most $k-1$. Let $r=\frac{|V|}{k}$. Notice that $r \geq 2$.

Let $C$ a complete graph of $l \cdot r$ vertices and let $G^{\prime}$ be the join of $G$ and $C$. We will show that $G^{\prime} \in(\boldsymbol{k}, \boldsymbol{l})_{\boldsymbol{E}}$ if and only if $G$ can be equitable colored with $k$ colors. If $G$ can be equitably colored with $k$ colors, then each color class of an equitable coloring of $G$ has exactly $r$ vertices. Hence we can partition the original vertices of $G^{\prime}$ into $k$ independent sets, and vertices from $C$ into the $l$ cliques of size $r$.

Conversely, if $G^{\prime} \in(k, l)_{E}$, then we must make the following observation: if a vertex from $V(C)$ belongs to one of the $k$ independent sets, then no other vertex of $G^{\prime}$ can belong to that independent set, since vertices of $V(C)$ are universal in $G^{\prime}$. Now, due to the fact that $|V(G)| \geq 2 k$, we have that $\left|V\left(G^{\prime}\right)\right| \geq 2(k+l)$, that is, each independent set has at least 2 vertices in any equitable partition. Therefore, none of the inserted vertices of $G^{\prime}$ belong to an independent set. So the original vertices of $G$ form the $k$ independent sets, that is, $G$ can be equitably colored with $k$ colors.

\section{References}

Brandstädt, A. (1984). Partitions of graphs into one or two independent sets and cliques. Technical report, Forschungsergebnisse der Friedrich-Schiller-Universitatät Jena.

Brandstädt, A. (1996). Partitions of graphs into one or two independent sets and cliques. Discrete Mathematics, 152(1-3):47-54.

Brandstädt, A. (1998). Corrigendum. Discrete Math., 186:295.

Karp, R. M. (1972). Reducibility among combinatorial problems. In Complexity of computer computations, pages 85-103. Springer. 